\title{
Stark broadening tables for the helium I 447.1 line
}

\section{Application to weakly coupled plasmas diagnostics ${ }^{\star}$}

\author{
M. A. Gigosos ${ }^{1}$ and M. Á. González ${ }^{2}$ \\ 1 Departamento de Óptica, Universidad de Valladolid, 44071 Valladolid, Spain \\ e-mail: gigosos@coyanza.opt.cie.uva.es \\ 2 Departamento de Física Aplicada, Universidad de Valladolid, 44071 Valladolid, Spain \\ e-mail: manuelgd@termo.uva.es
}

Received 1 April 2009 / Accepted 7 May 2009

\begin{abstract}
Context. Stark broadening of the 447.1 line of neutral helium in a weakly coupled plasma has been studied. This spectral line has a very close forbidden component. This means it is very sensitive to the plasma electron density, which is similar to hydrogen lines, for which the Stark effect is linear.

Aims. The aim of this work is to supply information to be used in the spectroscopic diagnostics of plasmas.

Methods. We used computer simulations in the frame of a physical model for a plasma with weak coupling between charges. The calculation includes the so-called "ion dynamics effects" in a natural way. The electron density, the ionic and electronic temperatures, and the perturber mass were the parameters in the calculations.

Results. Simulation results relate the line width, shift, shape, and especially the ratio of intensities between the allowed and forbidden components, with the plasma parameters. These results are supplied as tables of line shapes.
\end{abstract}

Key words. line: profiles - atomic data - plasmas

\section{Introduction}

The study of the hydrogen and helium spectral line shapes has supplied - and still does - a plasma diagnositics tool that is essential for analyzing star atmospheres and laboratory plasmas. Different characteristics of the measured lines are used to obtain the parameters of interest. In some cases the existence of a given line in the spectrum is already a valuable source of information. In others, the details of the line shape, such as its full width at half maximum $(F W H M)$, the peak shift, or the distance between the line peaks or the full line shape in the cases with satellite lines are used to know, for example, the plasma temperature, its electron density, or the existence of inhomogeneities, turbulences, or thermodynamic imbalances. For the case we are dealing with here, the $4471 \AA$ He I line is used in astronomy to obtain data for several stars. For example, in the bibliography one can find works that use this line simply to detect helium (Dufour et al. 2008). It is also used to measure the He abundances (Smart et al. 1996; Moehler et al. 2004; Huang \& Gies 2006), temperatures and surface gravity (Moehler et al. 2004), radial or rotational velocities (see, for example, (Levato et al. 1996; Kohoutek et al. 1999; Steele et al. 1999)), to do spectral classifications (Jeffery et al. 1997; Steele et al. 1999; Martayan et al. 2006; Bresolin et al. 2006; Vennes et al. 2007) or to measure the magnetic fields intensities (O'Toole et al. 2005). The

* Tables 2-11 are only available in electronic form at the CDS via anonymous ftp to cdsarc.u-strasbg.fr (130.79.128.5) or via http://cdsweb.u-strasbg.fr/cgi-bin/qcat?J/A+A/503/293 use of this line in laboratory plasmas is even more widespread (see the review Ivković et al. 2004, and the references therein).

In this work we have focused on the shape of the He I 4471 line due to the Stark broadening. Inside a plasma, the Stark effect produced by the local electric fields generated by the charged particles surrounding the emitter give rise to a line broadening that can easily be measured and related to the plasma parameters as the density of charged particles in the plasma, the plasma temperature, or its composition. This correspondence between the measured profiles and the plasma parameters has been used for a long time thanks to the numerous theoretical studies that have led to elaborate tables of line shapes with the electron density, the plasma particles temperature, or their masses as the input parameters (see the reviews of Griem 1974, 1997, and the plentiful bibliography cited there). These Stark profile tables cover a large set of many spectral lines of different elements. But the hydrogen and helium are the most studied elements, using both theoretical models and experimental measurements. Due to the greater ease of calculations, the hydrogen profiles have been a benchmark for the theoretical models. In the early seventies, there was already enough information to evaluate the theoretical models developed up to then and to know their limitations (Wiese et al. 1972, 1975). It is then that some theoretical treatments appeared that tried to avoid some of the simplifications that were considered, such as the consideration of quasistatic fields caused by the ions in the plasma. Several calculation methods were developed then and have proved to be very useful, such as the so-called model microfield method (MMM) (Frisch \& Brissaud 1971; Seidel 1977; 
Stehlé \& Hutcheon 1994), the frequency fluctuation method (FFM) (Talin et al. 1995), or the computer simulations (CS) (Stamm et al. 1984; Gigosos \& Cardeñoso 1987; Hegerfelt \& Kesting 1988; Gigosos \& Cardeñoso 1996). Then, these new treatments allowed one to face the so-called ion dynamics effects, which since the beginning were considered as the main reason for the observed discrepancies between the models established up to then and the experimental measurements (Wiese et al. 1975).

Hydrogen spectral lines are especially useful, not only because their calculation is easier (and more reliable, then), but also because the Stark effect is linear for that element, which gives rise to a large and easily measured line width. This situation also appears for other conditions (hydrogen-like lines with quasi-degenerate states) and in those configurations with satellite lines due to forbidden transitions. In particular, the $4921 \AA$ and $4471 \AA$ helium lines have been especially useful both as a test of theoretical models for laboratory plasmas and for their direct use in plasma diagnotics (Ivković et al. 2004), because in both cases one can measure the intensity of the forbidden line that can be as intense as, or even more intense than, the main allowed component but that is kept separate enough of the main component to be recorded as an independent spectral line. This line appears as result of the breaking off of the selection rule that impedes the transition between states with the same parity because the mixing of states caused by the Stark effect. This mixing, then, depends on the density of charged particles (which produce the local electric field), and its consequences can be considered as a probe of that density. The Stark effect affects both the ratio of intensities between the allowed and the forbidden components of the line and the spectral position of both components, so that this line can give information in two ways. Then, even for the plasma configurations where this line shows self-absorption and, then its Stark width is not useful for plasma diagnostics, the position of both peaks - allowed and forbidden - and their separation still give information on the electron density in the plasmas because their position is not affected by the opacity of the plasma (see comments by Valognes \& Bardet 1996). To these advantages of plasma diagnostics through the He 4471 line over hydrogen lines, one must add that the helium profiles are less sensitive to the distortive effects of the cold layers in laboratory plasmas because of the high ionization potential of helium.

In general, this line is considered in the bibliography as useful in plasma diagnostics such as the hydrogen lines, but, logically it has been studied less. Besides, some experimental works have focused on the ion dynamics effects to conclude that these effects are less than observed in some hydrogen lines, especially at high densities, where these effects were considered as irrelevant (Barnard et al. 1974; Greig et al. 1974) in spite of the low precision in determining the temperature of laboratory plasmas (see Valognes \& Bardet 1996). In this work we show that ion dynamics, as shown by the simulation results and by some characteristics of the line shapes, is more important than considered to now.

\section{Stark broadening model}

Since Anderson (Anderson 1949), the so-called standard theory (ST) (Sahal-Brechot 1969; Griem 1974, 1997), the MMM (Frisch \& Brissaud 1971; Seidel 1977; Stehlé \& Hutcheon 1994), the FFM (Talin et al. 1995, 1997), or the computer simulations (Stamm et al. 1984; Gigosos et al. 2003), just to give several of the mathematical treatments in this field, have considered that the line broadening due to the Stark effect is due to the action of the electric fields produced by the ions and electrons that surround each of the emitters in the plasma. In the formal treatment of this effect, the set of perturbers is considered as a thermal bath that alters the emission process of the atoms but that process does not modify the plasma behavior. In this way, the study of the emission is based on the statistical independence of the perturbers, on one hand, and the emitters, on the other. Besides, the emission processes of different atoms are considered to be incoherent. The profile calculation is carried forward, then, considering a typical emitter subjected to that thermal bath of the perturbers. The individual effect of that interaction is averaged considering the statistics over the possible states of the emitter, on the one hand, and over the possible actions of the perturbers, on the other. In the ST, these statistics consider the characteristic parameters of the collisions between the perturbers and the emitter as random variables - mainly impact parameter and perturber velocity. The MMM and the computer simulations use the time sequence of the perturber electric microfield as a random process whose statistical characteristics determine the shape of the spectral line. The ST, besides, considers in different way the actions due to the ions, which move slowly, and to the electrons which move quickly. The ionic field is considered in the framework of a model of a quasistatic field so that along the coherence time of the emission process, that field can be considered as static and gives rise to a Stark effect that remains constant during the emission process and that shifts the spectral lines. The joint effect of the ions in the plasma is determined then by the statistical distribution of that quasistatic field. In that theory, on the contrary, the perturbations due to the electrons are considered to be very quick so that they are studied in the framework of an impact model. The drawback of this treatment is that it does not properly consider the intermediate conditions, such as the so-called ion dynamics effects due to the ions, because the ionic field is not stationary. On the other hand, the simulation methods, as well as the MMM, consider the ionic and electronic fields on the same foot, so that they can consider those dynamic effects in a natural way ${ }^{1}$.

In all the treatments, the emission profile is obtained by the Fourier transform of the autocorrelation function of the emitter dipole moment:

$$
\begin{aligned}
& I(\Delta \omega)=\operatorname{Re} \frac{1}{\pi} \int_{0}^{\infty} \mathrm{d} t\{C(t)\} \mathrm{e}^{\mathrm{i} \Delta \omega t} \\
& C(t)=\operatorname{tr}[\boldsymbol{D}(t) \cdot \boldsymbol{D}(0) \rho] \\
& \boldsymbol{D}(t)=U^{+}(t) \boldsymbol{D}(0) U(t)
\end{aligned}
$$

where $U(t)$ is the emitter time evolution operator, that obeys the Schrödinger's equation

$\mathrm{i} \hbar \frac{\mathrm{d}}{\mathrm{d} t} U(t)=H(t) U(t)=\left(H_{0}+q \boldsymbol{E}(t) \cdot \boldsymbol{R}\right) U(t)$,

where the Hamiltonian $H(t)$ includes the structure of the emitter states without perturbation, $H_{0}$, and the action of the perturbers through the dipole interaction with the electric field $\boldsymbol{E}(t)$ at the emitter position. In Eq. (1), the symbols \{\} mean an average over the individual processes. On the one hand, the average over all the possible states of the emitter is carried forward through the trace of the matrix that appears in Eq. (2), considering the emitter's possible states, weighted using the density matrix $\rho$. On the

\footnotetext{
1 The model of Barnard et al. (1974), mentioned above and whose results will be used as a reference in the next sections include in an approximate way those effects, but only for the cases of low electron density.
} 


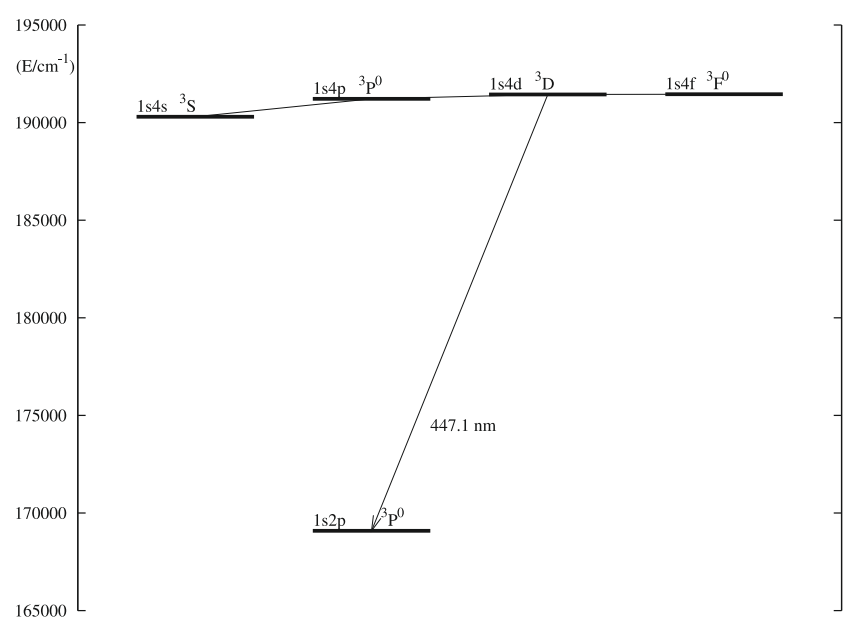

Fig. 1. Diagram of the levels involved in the transition studied in this work.

other hand, that average requires to consider different perturbing sequences $\boldsymbol{E}(t)$. As already said, the ST treatment separates that perturber field into two statistically independent parts. The first, the ionic field, is considered as static, so that it is averaged considering a known field distribution (see, for example, Hooper 1968; or Iglesias \& Lebowitz 1984). The electronic field is considered to be formed as a sequence of non-overlapped individual collisions with a very short length in the typical duration of the emission process. This sequence is statistically analyzed, as said before, taking the collision parameters as random variables.

In general, in all the models, including the one used in the computer simulation shown in this work, one considers that the emission process is described with enough accuracy when only taking a finite set of quantum states into account. Then, two groups of states are considered: the states "of the upper level" and those of the "lower level", formed by those involved in the transition under study, together with those that, due to the perturbers' action, are connected to them by the Stark effect. These groups, upper and lower, in the optical domain are separated enough in energies that one can consider that the plasma perturbations do not induce transitions between them. Within this condition one uses the "no-quenching approximation".

Figure 1 shows the transitions' diagram considered in this study of the He I $447.1 \mathrm{~nm}$ line. The upper group has 16 states (spin plays no role in our calculation), and the lower one 3 . The 447.1 line is emitted as a transition between the $1 \mathrm{~s} 4 \mathrm{~d}^{3} \mathrm{D}$ and the $1 \mathrm{~s} 2 \mathrm{p}^{3} \mathrm{P}^{\mathrm{o}}$ states. The most important forbidden line appears because a transition due to the Stark perturbed level $1 \mathrm{~s} 4 \mathrm{f}^{3} \mathrm{~F}^{\mathrm{o}}$ and the same of the lower group. At very high density a second forbidden line can be observed because of a transition from the $1 \mathrm{~s} 4 \mathrm{p}^{3} \mathrm{P}^{\mathrm{o}}$ level.

\section{Physical model for the plasma in the computer simulation}

Computer simulations, especially those used in this work, obtain the emission profile from the Eqs. (1) to (4) averaging over a representative set of field sequences $\boldsymbol{E}(t)$ calculated, all of them, through a molecular dynamics numerical calculation carried forward in a computer.

We used a spherical volume that contains $N$ ions and $N$ electrons $(N=500$ in this work) randomly placed and moving independently following straight-line trajectories with constant speed. The speeds of those particles obey the Maxwell distribution according to the equilibrium temperature of each of the species and a mass equal to the reduced mass of the emitterperturber pair ( $\mu$-ion model (Seidel \& Stamm 1982)). When one of those particles reaches the border of the simulation sphere, it is replaced by another particle so that the density of particles in the sphere is kept constant. The reinjection technique guarantees that both the spatial distribution of particles and the velocities distribution are kept stationary and that no correlation effects between the exiting and the incoming particles appear. See the reference Gigosos \& Cardeñoso (1996) for a detailed explanation of this method.

The emitter atom is placed in the center of the simulation sphere, and it undergoes the action of the electric fields produced by all the charged particles. To calculate that field, the Debye screened field expression is used, so that the correlation effects between the charged particles in the plasma can be considered, at least approximately. This treatment is limited, then, to weakly coupled plasmas, such as those considered here. Those cases with high values for the coupling parameter that were nonetheless included in the figures shown in this work, must be considered as extrapolations of this method (see Dufour et al. 2005, for a discussion of this approximation).

The electric field time sequence obtained in this way is used to calculate an autocorrelation function of the emitter's dipole moment using the Eqs. (2) and (3) from a numerical solution of the differential Eqs. (4) that give the emitter's time-evolution operator. The process is repeated to obtain a representative sampling of field $\boldsymbol{E}(t)$ sequences (in this work, between 20000 and 30000 samples, depending of the studied case). The average of all the autocorrelation functions obtained from these field sequences $\{C(t)\}$ is used to obtain the spectral profile through its Fourier transform.

\section{Numerical treatment in the profile calculation}

In the simulation process, the movement of the particles is described by discrete time steps of a given size $\Delta t$. This size is chosen so that the dynamic evolution of the field is described in enough detail. Besides, the step must be small enough so that the differential Eqs. (4) can be solved with enough accuracy. The first requirement is, in fact, stronger than the second one. Then, in our treatment, we chose the time step $\Delta t$ small enough that the electric field can be considered static within it. In this way, we write

$$
\begin{aligned}
U(t+\Delta t) & =M(t+\Delta t, t) U(t) \\
& \simeq \exp \left[-\frac{\mathrm{i}}{\hbar}\left(H_{0}+q \mathbf{E}(t) \cdot \mathbf{R}\right) \Delta t\right] U(t)
\end{aligned}
$$

Our treatment was done with $\Delta t=0.01 t_{0}$, where $t_{0}=r_{0} / v_{0}$ the electrons dynamics characteristic time $-r_{0}=\left(3 /\left(4 \pi N_{\mathrm{e}}\right)\right)^{1 / 3}$ and $v_{0}=\sqrt{2 k T_{\mathrm{e}} / m_{\mathrm{e}}}$ are the "natural" interparticle distance and velocity given by the electron density $N_{\mathrm{e}}$ and temperature $T_{\mathrm{e}}-$. We checked that a narrower time step gives the same result within the simulation's statistical noise.

The matrices appearing in (5) are, in our case, of size $16 \times$ 16. To obtain the exponential that appears in that expression, the diagonalization algorithm known as "Jacobi's method" was used (Press et al. 1992). The matrices are calculated in the socalled "cartesian base of states", so that all their elements are real numbers, which notably simplifies their numerical treatment. Table 1 gives the physical values used in $H_{0}$, the non-perturbed Hamiltonian, and in the emitter dipolar moment operator, $q \boldsymbol{R}$. 
Table 1. Data of the helium I atomic structure used in this work.

\begin{tabular}{ccccc}
\hline \hline Config. & Term & $E_{i}{ }^{*}$ & $E_{k}$ & $S_{i k}$ \\
\hline $1 \mathrm{~s} 4 \mathrm{~s}-1 \mathrm{~s} 4 \mathrm{p}$ & ${ }^{3} \mathrm{~S}-{ }^{3} \mathrm{P}^{\mathrm{o}}$ & 190298.11 & 191217.06 & $1.30 \mathrm{e}+03$ \\
$1 \mathrm{~s} 4 \mathrm{p}-1 \mathrm{~s} 4 \mathrm{~d}$ & ${ }^{3} \mathrm{P}^{\mathrm{o}} \mathrm{B}^{3} \mathrm{D}$ & 191217.06 & 191444.48 & $2.61 \mathrm{e}+03$ \\
$1 \mathrm{~s} 4 \mathrm{~d}-1 \mathrm{~s} 4 \mathrm{f}$ & ${ }^{3} \mathrm{D}-{ }^{3} \mathrm{~F}^{\mathrm{o}}$ & 191444.48 & 191451.88 & $2.09 \mathrm{e}+03$ \\
\hline
\end{tabular}

* The energies $E_{i}$ and $E_{k}$ are given in $\mathrm{cm}^{-1}$ and $S_{i k}$ in atomic units. These data have been taken from http://physics.nist.gov/ PhysRefData/ASD/lines_form.html.

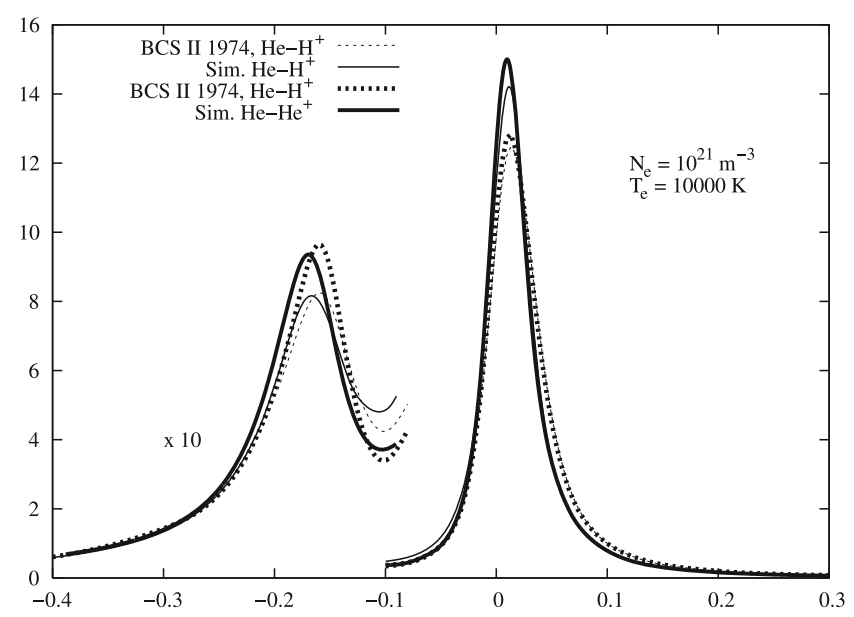

Fig. 2. Comparison of the simulation results with those of Barnard et al. (1974) at low density, with Doppler broadening included in all cases.

\section{Results}

The results of this work are given in tables of profiles for their use in plasma diagnostics. Those tables supply the profile $I(\Delta \lambda)$ area-normalized so that $\int I(\lambda) \mathrm{d} \lambda=1$, where $\Delta \lambda=\lambda-\lambda_{0}$ and $\lambda_{0}$ the wavelength of the transition between the states $1 \mathrm{~s} 4 \mathrm{~d}^{3} \mathrm{D}$ and $1 \mathrm{~s} 2 \mathrm{p}{ }^{3} \mathrm{P}^{\mathrm{o}}$ (measured in air: $447.1479 \mathrm{~nm}$ ). In this section we point out some of the more relevant charateristics of this line. Our aim here is not to do a fully detailed comparison between our results and experimental data as will be done in a more complete study. Here, only some comparison of reference will be shown and propose the reader to look through the wide available bibliography on this subject ${ }^{2}$.

Figure 2 shows a first comparison between our simulation and the model of Barnard et al. (1974) at low density. The results are similar, though the simulation widths are a little lower. Figures 3 and 4 compare results for higher densities. The data of Griem (1968) and simulations of Calisti et al. (1988) have also been included. From $N_{\mathrm{e}}=10^{22} \mathrm{~m}^{-3}$ on, the authors of Barnard et al. (1974) consider that ion dynamics has no effect, which does not completely agree with the simulation results.

Figure 5 shows a general comparison of the results for low and medium densities. The image of the classical work of Kelleher (1981) was employed. In that figure the results of the simulation were overimposed after normalization so that the

\footnotetext{
2 See the "reviews" of Ivković et al. (2004) and Lesage (2008), where the most relevant works are commented and compared with the theoretical models. The work of Czernichowski \& Chapelle (1985) can also be seen, as the authors of that group join their results with those of other authors and supply empirical formulas that can be used as a reference. In Valognes \& Bardet (1996) theoretical results are also compared with the most interesting data in the bibliography.
}

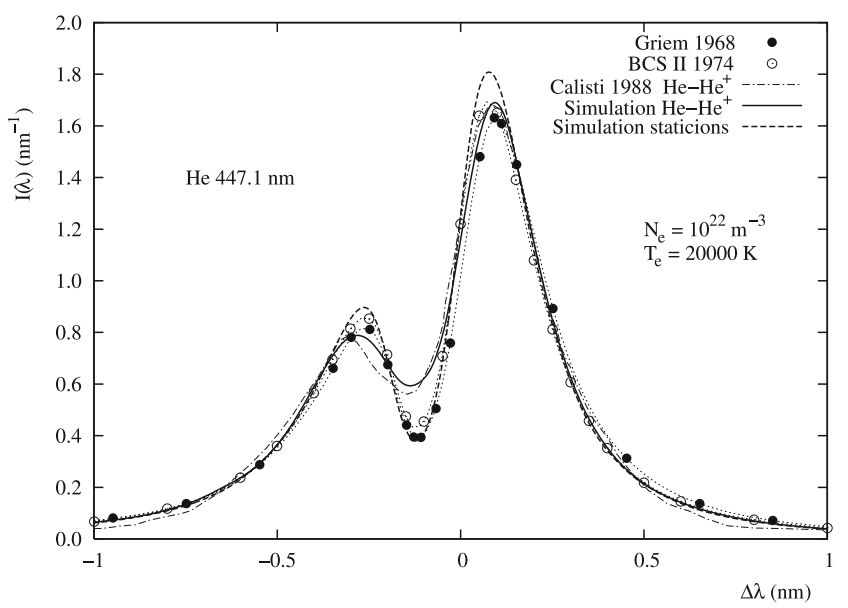

Fig. 3. Comparison models vs. simulations. Doppler broadening has been included in all cases.

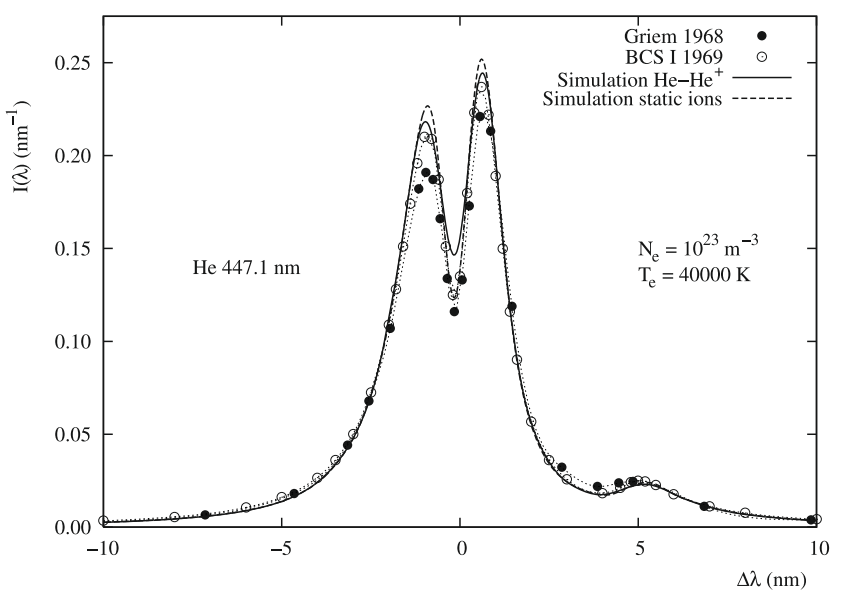

Fig. 4. Comparison models vs. simulations at high density.

intensities of the allowed transition peaks in the experiment and in the simulation coincide. For the calculation, the values of electron density given by the author of the experiment in the same figure were used and the temperatures 20000, 18000, 15000, 12000 , and $10000 \mathrm{~K}$ from higher to lower density, respectively. The calculation profiles were scaled so that the allowed peak intensity corresponds to that of the experiment, but the position of the line has not been changed. As can be seen here the shift of the allowed component peak agrees very well with the experimental results.

Figure 6 shows the parameters usually employed to characterize the shape of this spectral line. Below, we show their dependences with the plasma parameters according to our simulation results.

The full width at half maximum $(F W H M)$ is one of the parameters useful to do plasma diagnostics when the line selfabsorption is not a problem. At low density, when the forbidden component has a low intensity and both components can be clearly distinguished, the allowed component width follows behavior typical of the impact broadening; that is, the line width is nearly linear with the electron density. A logarithmical fitting between the total width and the electron density of the type $\log F W H M=a+b \log N_{\mathrm{e}}$, gives values for the parameter $b$ between 0.919 and 0.896 for values of $N_{\mathrm{e}}$ between $10^{21}$ and $10^{22} \mathrm{~m}^{-3}$ and temperatures between 5000 and $40000 \mathrm{~K}$. For higher densities, when both components are overlapped, the 


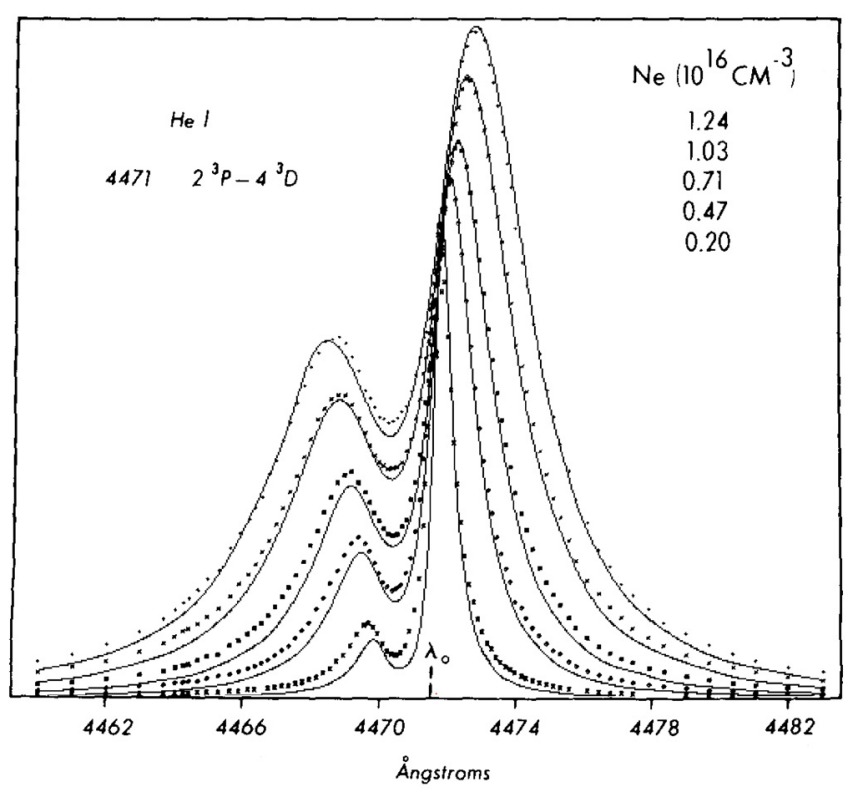

Fig. 5. Comparison simulation vs. experiment. The experimental data are a reproduction of Fig. 5 in the classical work by Kelleher (1981) (with permission of the author).

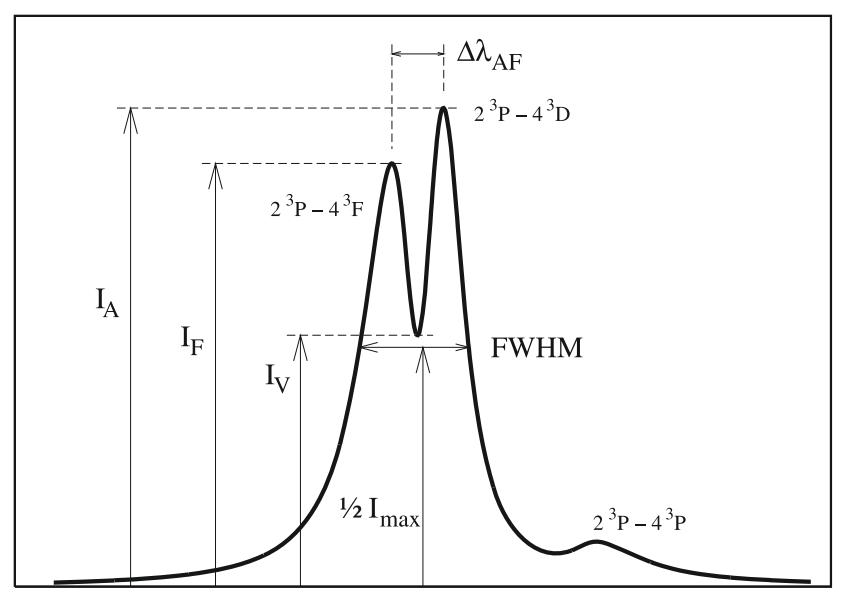

Fig. 6. Picture showing the 447.1 line parameters that have been analyzed in this work.

quasistatic effects due to the ionic field begin to be noticeable and for that same fitting the value of the parameter $b$ changes between 0.670 at $5000 \mathrm{~K}$ and 0.762 at $40000 \mathrm{~K}$, close to the value of $2 / 3$ typical of the quasistatic model for the transitions with linear Stark effect (see Fig. 7). This behavior is coherent with the dependence of the width with the temperature, as can be seen in the Fig. 8.

The most interesting detail of the transition under study is, perhaps, the separation between the allowed and forbidden peaks. As already said, this parameter is useful in plasma diagnostics because it is strongly affected neither by the selfabsorption nor by the Doppler effect and because its dependence on the plasma electron density is very clear. Figure 9 shows this trend. Data corresponding to different temperatures for a pure helium plasma have been represented together. This parameter is completely insensitive to the ion dynamics effects, so that the same results are obtained for other values of $\mu$. It must be pointed out how the simulation data for a temperature of $5000 \mathrm{~K}$ coincide with the empirical formula of Czernichowski \& Chapelle (1985)

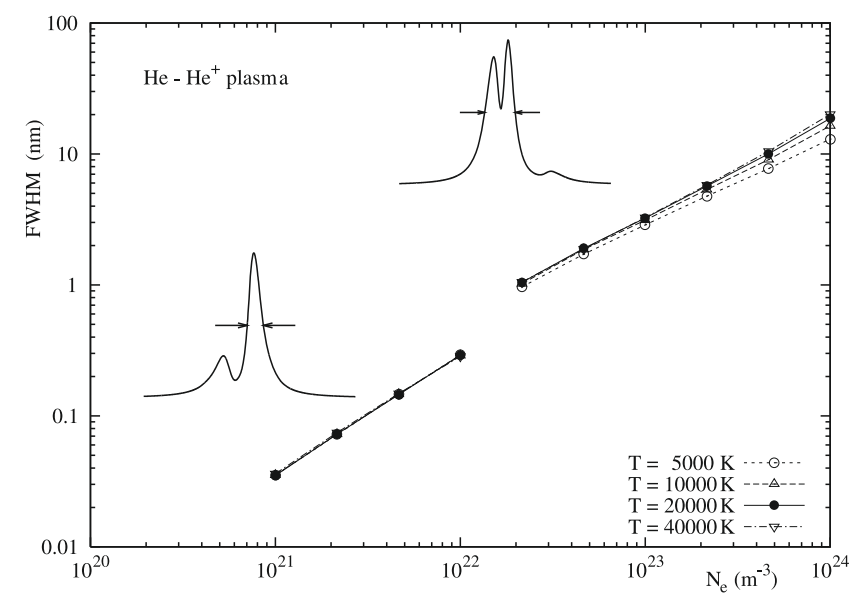

Fig. 7. Full width at half maximum of the $447.1 \mathrm{~nm}$ He I line. The represented values correspond to $\mu=2.0$. At low densities, the line width is nearly independent of the temperature.

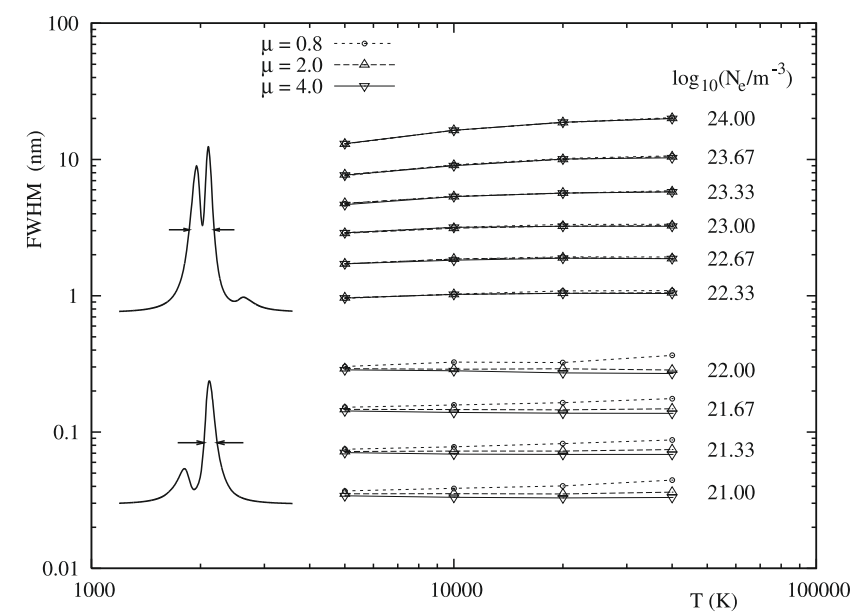

Fig. 8. Full width at half maximum $(F W H M)$ of the $447.1 \mathrm{~nm}$ He I line as a function of the electronic temperature. As can be seen, the influence of the perturber mass is minimal at high densities and relatively weak at low densities, when both components of the profile are not overlapped.

obtained fitting experimental data with densities between $\approx 8 \times$ $10^{20} \mathrm{~m}^{-3}$ and $\approx 2 \times 10^{22} \mathrm{~m}^{-3}$ and temperatures around $10000 \mathrm{~K}$. In the following, the fittings of these authors are used as a reference, just because in their work many bibliographic data were compared and its use in plasma diagnostics using line shape data proposed.

Figure 10 shows the results of one of the line parameters useful in plasma diagnostics: the ratio between the intensities of the allowed and forbidden components peaks as a function of the electron density in the plasma. This ratio is nearly independent of the ion dynamics effects as can be seen from the simulation results. A comparison between simulation results and the curves of Czernichowski \& Chapelle (1985), obtained fitting experimental data, is shown in Fig. 11. The correspondence with experimental data is good, but an extrapolation of the empirical formula of Czernichowski \& Chapelle (1985) is out of place. As happened with Fig. 9, it is noticeable that the simulation results correspond better with the experimental fittings for low temperatures (see Fig. 4 in the original work of Czernichowski \& Chapelle 1985, to localize the experimental data they compiled).

The last parameter considered here is the ratio between the intensity of the minimum at the valley between the two peaks 


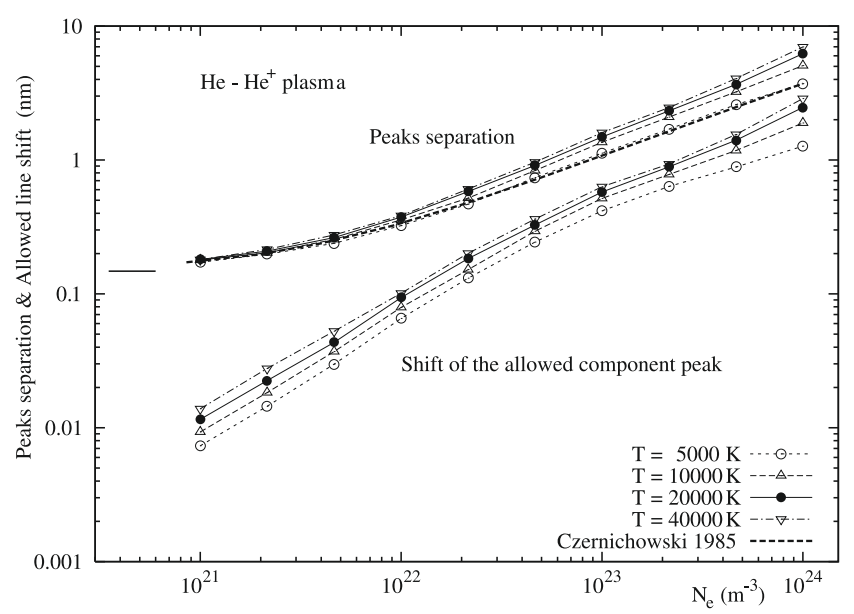

Fig. 9. Separation of the allowed and forbidden transitions peaks and shift in the allowed transition as a function of the plasma electron density. Data corresponding to different temperatures for a pure helium plasma $(\mu=2.0)$ are shown together. The horizontal line marks the peaks distance corresponding to the distance in energies of the levels $4{ }^{3} \mathrm{~F}$ and $4{ }^{3} \mathrm{D}$.

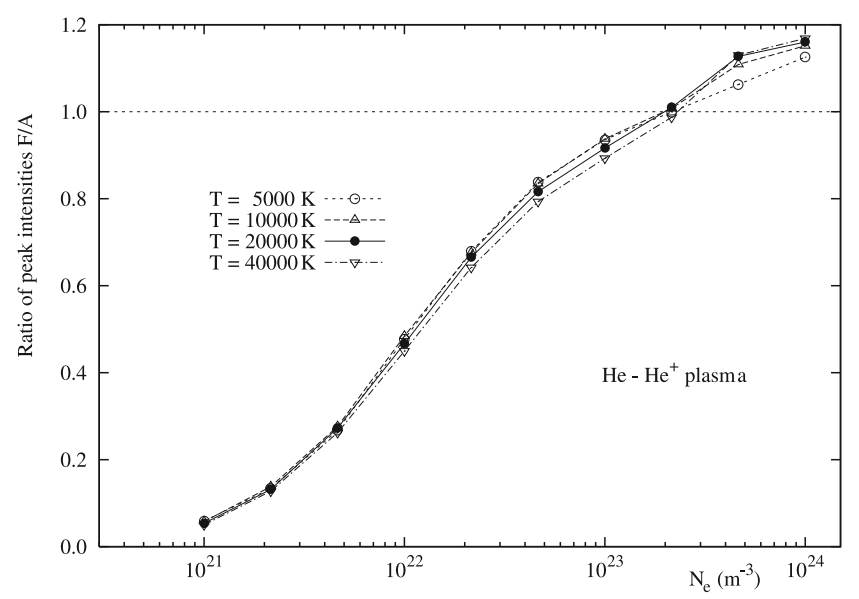

Fig. 10. Ratio $I_{\mathrm{F}} / I_{\mathrm{A}}$ of the forbidden and allowed peak intensities as a function of the plasma electron density. Data corresponding to different temperatures for a pure helium plasma $(\mu=2.0)$ are shown together.

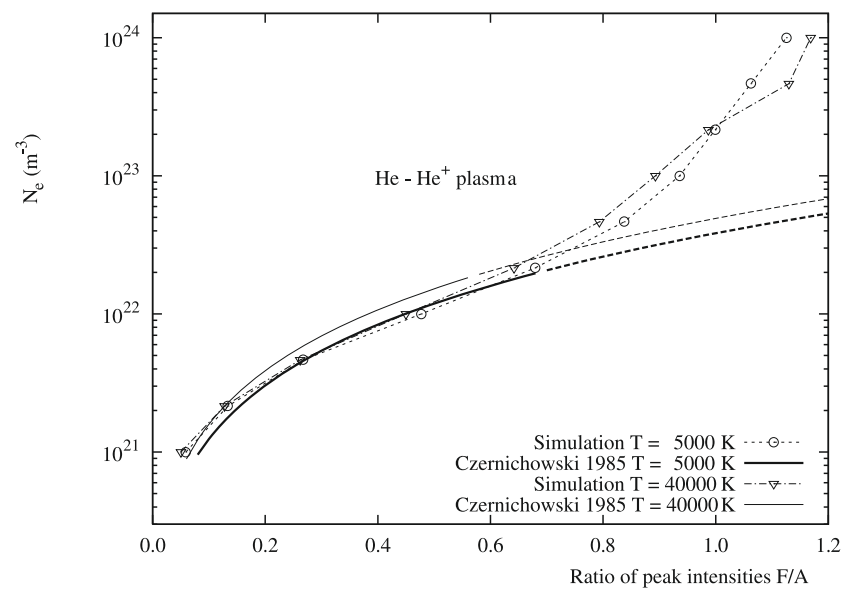

Fig. 11. Diagnostics curves for the electron density as a function of the ratio $I_{\mathrm{F}} / I_{\mathrm{A}}$ of the forbidden and allowed component peak intensities. The dashed curve gives the extrapolation of that fitting to larger densities than those considered by the authors of that work.

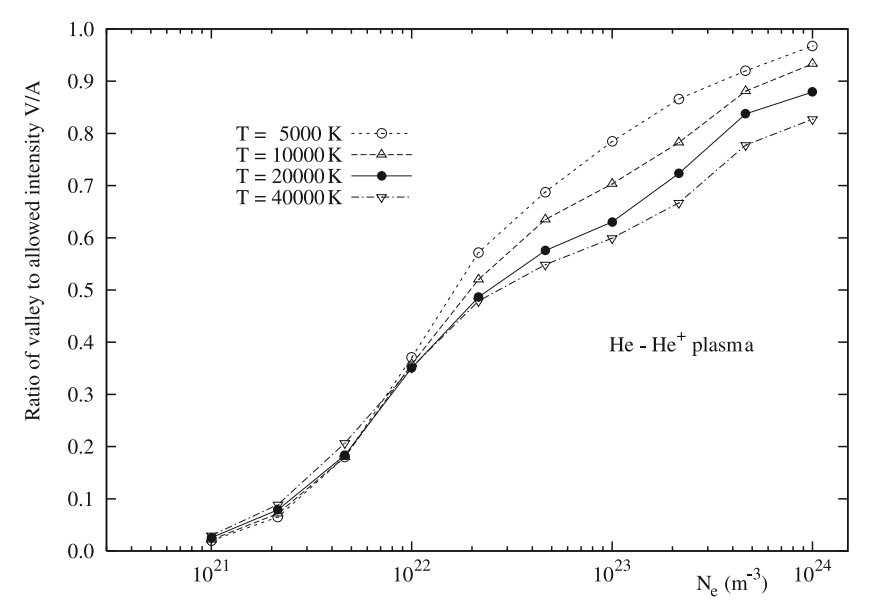

Fig. 12. Ratio of the intensity of the valley between the two peaks to the intensity of the allowed line as a function of the plasma electron density. As can be seen here, the influence of the temperature on the value of this parameter is quite noticeable.

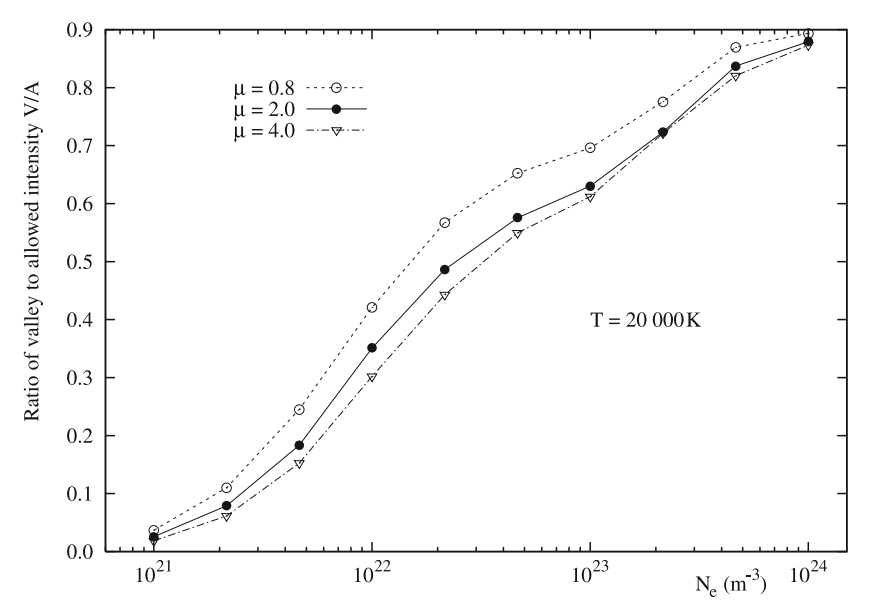

Fig. 13. Ratio $I_{\mathrm{V}} / I_{\mathrm{A}}$ of the valley between the two peaks to the allowed line peak as function of the electron density. Data for different reduced masses (corresponding to $\mathrm{He}-\mathrm{H}^{+}$, pure helium and helium with much heavier perturbers) are represented here.

and the allowed peak intensity. This is the most depending parameter on ion-dynamics effects and on the electronic temperature. Figures 12 and 13 show this statetment. As in the former case, Czernichowski \& Chapelle (1985) supplies a fitting to be used in plasma diagnostics and calculated from experimental results. Figure 14 shows a comparison of that empirical formula with the results of our simulation. As could also be observed in Fig. 11, an extrapolation of that empirical formula is not convenient. The influence of the temperature on the values of the represented parameter obtained in the simulations is much greater than estimated by the authors of that fitting. One must take into account that determinating the temperature is one of the difficulties in the experiments (Valognes \& Bardet 1996).

We show the results in Fig. 15, which has been taken as a reference in many works in the bibliography (Calisti et al. 1988; Schöning 1994; Ivković et al. 2004): the comparison between the results of Richter \& Piel (1985) and those obtained in our simulations. This figure shows the most relevant aspect of the 


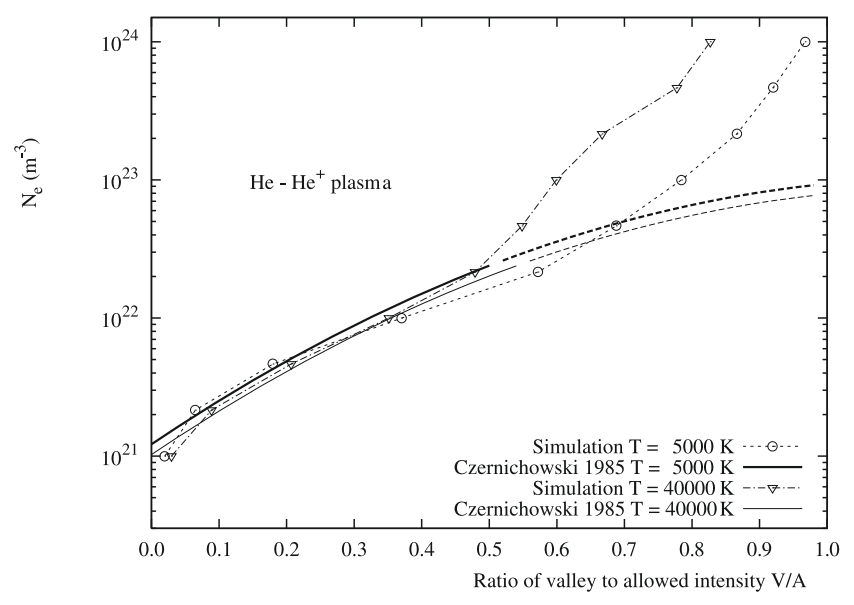

Fig. 14. Diagnostics curves for the electron density as a function of the ratio $I_{\mathrm{V}} / I_{\mathrm{A}}$ of the valley between the two peaks to the allowed transition intensity. Data for the two extreme temperatures considered in this work are shown in the figure for a pure helium plasma $(\mu=2.0)$. The curves given in Czernichowski \& Chapelle (1985) were obtained as a fitting of experimental data. An extrapolation of that fitting to values of electron density higher than those considered by the authors of that work is shown with a dashed line.

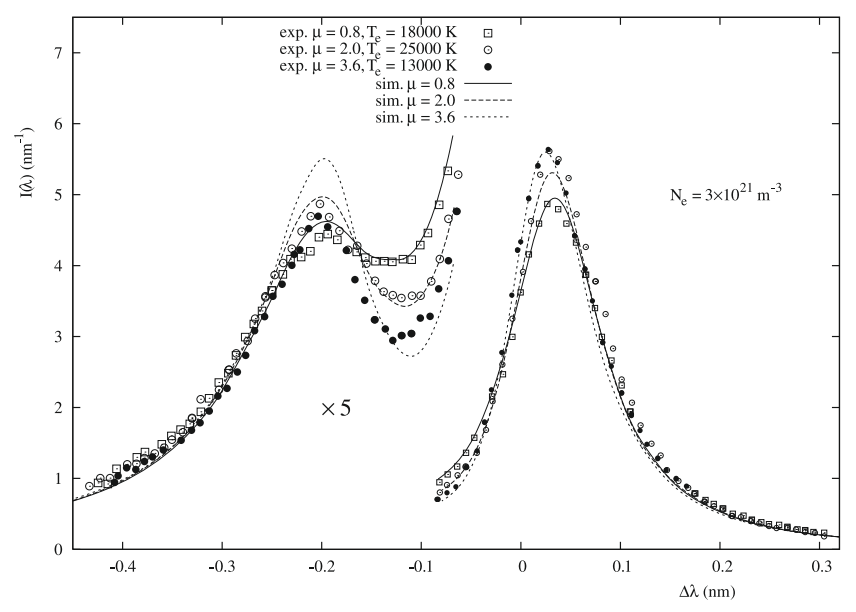

Fig. 15. Comparison between simulation results and the experimental data of Richter \& Piel (1985).

ion dynmics effects. As in Fig. 5, a good correspondence between theory and experiment can be observed, which makes one confident of the usefulness of the diagnostics tables shown in this work.

Acknowledgements. This work has been partially financed by Spanish Ministerio de Educación y Ciencia through grant ENE2007-63386/FTN and by the Junta de Castilla y León through grant VA032A06.

\section{References}

Anderson, P. W. 1949, Phys. Rev., 76, 647

Bardet, J. P., Valognes, J. C., \& Vitel, Y. 2000, J. Quant. Spectrosc. Radiat. Transfer, 65, 853

Barnard, A. J., Cooper, J., \& Shamey, L. J. 1969, A\&A, 1, 28
Barnard, A. J., Cooper, J., \& Smith, E. W. 1974, J. Quant. Spectrosc. Radiat. Transfer, 14,1025

Birkeland, J. W., Bacon, M. E., \& Braun, W. G. 1971, Phys. Rev. A, 3, 354

Bresolin, F., Pietrzyński, G., Urbaneja, M. A., et al. 2006, ApJ, 648, 1007

Calisti, A., Stamm, R., \& Talin, B. 1988, Phys. Rev. A, 38, 4883

Collins, G. W., Truax, R. J., \& Cranmer, S. R. 1991, ApJS, 77, 541

Czernichowski, A., \& Chapelle, J. 1985, J. Quant. Spectrosc. Radiat. Transfer, 33, 427

Dufour, E., Calisti, A., Talin, B., et al. 2005, Phys. Rev. E, 71, 066409

Dufour, P., Fontaine, G., Liebert, J., Williams, K., \& Lai, D. K. 2008, ApJ, 683, L167

Fleurier, C., Couland, G., \& Chapelle, J. 1978, Phys. Rev. A, 18, 575

Frisch, U., \& Brissaud, A. 1971, J. Quant. Spectrosc. Radiat. Transfer, 11, 1753 and 1767

Gavrilenko, V. P., Kyrie, N. P., \& Frank, A. G. 2003, Plasma Phys. Rep., 29, 443 Gigosos, M. A., \& Cardeñoso, V. 1987, J. Phys. B: At. Mol. Opt. Phys., 20, 6005 Gigosos, M. A., \& Cardeñoso, V. 1996, J. Phys. B: At. Mol. Opt. Phys., 29, 4795 Gigosos, M. A., González, M. Á., \& Cardeñoso, V. 2003, Spectrochimica Acta Part B, 58, 1489

Greig, J. R., Jones, L. A., \& Lee, R. W. 1974, Phys. Rev. A, 9,44

Griem, H. R. 1968, ApJ, 154, 1111

Griem, H. R. 1974, Spectral Line Broadening by Plasmas (New York: Academic Press)

Griem, H. R. 1997, Principles of plasma spectroscopy (Cambridge, UK: Cambridge University Press)

Hegerfeldt, G. C., \& Kesting, V. 1988, Phys. Rev. A, 37, 1488

Hooper, C. F. 1968, Phys. Rev., 169, 193

Huang, W., \& Gies, D. R. 2006, ApJ, 648, 591

Iglesias, C. A., \& Lebowitz, J. 1984, Phys. Rev. A, 30, 2001

Ivković, M., Jovicévić, S., \& Konjević, N. 2004, Spectrochimica Acta Part B, 59,591

Jeffery, C. S., Drilling, J. S., Harrison, P. M., Hebert, U., \& Moehler, S. 1997, A\&AS, 125, 501

Kelleher, D. E. 1981, J. Quant. Spectrosc. Radiat. Transfer, 25, 191

Kohoutek, L., Mayer, P., \& Lorenz, R. 1999, A\&AS, 134, 129

Lesage, A. 2009, New Astron. Rev., 52, 471

Levato, H., Malaroda, S., Morrell, N., Solivella, G., \& Grosso, M. 1996, A\&AS, 118,231

Martayan, C., Frémat, Y., Hubert, A.-M., et al. 2006, A\&A, 452, 273

Mijatović, Z., Kobilarov, R., Djurocić, S., \& Stevanov, M. 1997, Appl. Spectrosc., 51, 396

Milosavljević, V., \& Djeniže, S. 2001, Eur. Phys. J. D, 15, 99

Moehler, S., Sweigart, A. V., Landsman, W. B., Hammer, N. J. \& Dreizler, S. 2004, A\&A, 415, 313

O’Toole, S. J., Jordan, S., Friedrich, S., \& Heber, U. 2005, A\&A, 437, 227

Pérez, C., de la Rosa, I., Aparicio, J. A., Mar, S., \& Gigosos, M. A. 1996, Jpn. J. Appl. Phys., 35, 4073

Press, W. H., Teukolsky, S. A., Veterling, W. T., \& Flannery, B. P. 1992, Numerical Recipes in C (Cambridge, N.Y.: University Press)

Richter, H., \& Piel, A. 1985, J. Quant. Spectrosc. Radiat. Transfer, 33, 615

Sahal-Brechot, S. 1969, A\&A, 1, 91

Seidel, J. 1977, Z. Naturforsch, 32a, 1195 and 1207

Seidel, J., \& Stamm, R. 1982, J. Quant. Spectrosc. Radiat. Transfer, 27, 499

Schöning, T. 1994, J. Phys. B: At. Mol. Opt. Phys., 27, 4501

Smartt, S. J., Dufton, P. L., \& Rolleston, W. R. J. 1996, A\&AS, 116, 483

Stamm, R., Smith, E. W., \& Talin, B. 1984, Phys. Rev. A, 30, 2039

Steele, I. A., Negueruela, I., \& Clark, J. S. 1999, A\&AS, 137, 147

Stehlé, C., \& Hutcheon, R. 1999, A\&AS, 140, 93

Suemitsu, H., Iwaki, K., Takemoto, Y., \& Yoshida, E. 1990, J. Phys. B: At. Mol. Opt. Phys., 23, 1129

Talin, B., Calisti, A., Godbert, L., et al. 1995, Phys. Rev. A, 51, 1918

Talin, B., Calisti, A., Ferri, S., et al. 1997, J. Quant. Spectrosc. Radiat. Transfer, 58,953

Uzelac, N. I., \& Konjević, N. 1986, Phys. Rev. A, 33, 1349

Uzelac, N. I., Stefanović, I., \& Konjević, N. 1991, J. Quant. Spectrosc. Radiat. Transfer, 46, 447

Valognes, J. C., \& Bardet, J. P. 1996, J. Quant. Spectrosc. Radiat. Transfer, 56, 855

Vennes, S., Kawka, A., \& Smith, J. A. 2007, ApJ, 668, L59

Wiese, W. L., Kelleher, D. E., \& Paquette, D. R. 1972, Phys. Rev A, 6, 1132

Wiese, W. L., Kelleher, D. E., \& Helbig, V. 1975, Phys. Rev A, 11, 1854 